\title{
Screening Positive for Cognitive Impairment: Impact on Healthcare Utilization and Provider Action in Primary and Specialty Care Practices
}

\author{
Michael Rosenbloom, MD ${ }^{1,2}$, Terry R. Barclay, PhD ${ }^{1,2}$, soo Borson, MD ${ }^{3,4}$, Ann M. Werner, PhD'2, \\ Lauren O. Erickson, $M S^{2}$, Jean M. Crow, MA ${ }^{1,2}$, Kamakshi Lakshminarayan, $M D, P h D^{4}$, \\ Logan H. Stuck, PhD' ${ }^{2}$, and Leah R. Hanson, $P h D^{1,2}$
}

\footnotetext{
'Health Partners Center for Memory and Aging, St. Paul, MN, USA; ${ }^{2}$ HealthPartners Institute, Minneapolis, MN, USA; ${ }^{3}$ Department of Psychiatry and Behavioral Sciences, University of WA School of Medicine, Seattle, WA, USA; ${ }^{4}$ Neurology Department, University of MN, Minneapolis, MN, USA.
}

\begin{abstract}
BACKGROUND: Alzheimer's disease, the most common cause of dementia, goes unrecognized in half of patients presenting to healthcare providers and is associated with increased acute care utilization. Routine cognitive screening of older adults in healthcare settings could improve rates of dementia diagnosis and patterns of healthcare utilization.

OBJECTIVE: To evaluate the impact of screening positive for cognitive impairment on provider action in primary and specialty care practices and patient healthcare utilization.

DESIGN: Individuals asymptomatic for cognitive impairment completed cognitive screening with the Mini-Cog (MC). Outcomes included MC screen-positive rates, provider follow-up actions, and healthcare utilization for all participants over a period of 36 months (18 months prior to and following $\mathrm{MC}$ screening). Data were extracted from the electronic medical record (EMR). Healthcare provider interventions and healthcare utilization for screenpositive and -negative groups, before and after screening, were compared.
\end{abstract}

PARTICIPANTS: Primary and specialty care patients ( $n=$ 787) aged $\geq 65$ without history of cognitive impairment seen in HealthPartners, an integrated healthcare system in Minnesota and Western Wisconsin.

KEY RESULTS: In primary care and neurology practices combined, over the entire 36-month study window, individuals screening positive showed $32 \%$ higher rates of ED visits $(p<0.05)$ pre and post-screening compared to those screening negative. Screen positive also showed 39\% higher rates of hospitalizations pre-screening $(p<0.05)$ and 58\% higher rates post-screening $(p<0.01)$. While screen-detected cognitive impairment was associated with some relevant provider follow-up action in $32 \%$ of individuals, subsequent healthcare utilization did not

Research reported in this publication was supported by the National Center for Advancing Translational Sciences of the National Institutes of Health Award Number UL1TR000114

Electronic supplementary material The online version of this article (https://doi.org/10.1007/s11606-018-4606-4) contains supplementary material, which is available to authorized users.

Received January 4, 2018

Revised May 22, 2018

Accepted July 10, 2018

Published online August 10, 2018 change between the 18-month pre- and post-screening periods.

CONCLUSION: Despite being associated with higher rates of healthcare utilization, screening positive on the MC led to a change in provider action in a minority of cases and did not reduce post-screening healthcare utilization. Screening for cognitive impairment alone is not sufficient to alter patterns of provider practice or patient healthcare utilization.

KEY WORDS: dementia; aging; physician behavior; diagnosis.

J Gen Intern Med 33(10): 1746-51

DOI: $10.1007 / \mathrm{s} 11606-018-4606-4$

(C) Society of General Internal Medicine 2018

\section{INTRODUCTION}

Alzheimer's disease (AD) is the most common cause of dementia, affecting 5.5 million individuals in the USA with an estimated total societal cost as high as $\$ 259$ billion annually. ${ }^{1}$ Despite the fact that the number of cases is expected to triple by 2050 , a community cohort study showed that cognitive impairment goes unrecognized in 50\% or more of patients aged $\geq 70 .^{2}$ Consequently, the disease is most often recognized only in the later stages when key functions such as driving, financial management, and medication administration have already been compromised. Potential challenges relating to the diagnosis of Alzheimer's and related dementias (ADRD) include the lack of a single definitive diagnostic test, nihilism relating to absence of disease-modifying drugs, and reluctance of healthcare providers to share a terminal diagnosis with patients and families who may be emotionally unprepared for such news. ${ }^{3}$

The National Alzheimer's Plan has prioritized early detection of cognitive impairment and ADRD are also increasingly recognized as major drivers of impaired function, poor quality of life, poor adherence to medical treatment, and increased medical care utilization and healthcare costs. ${ }^{4}$ However, the US Preventive Services Task Force (USPSTF) has recommended against routine asymptomatic cognitive screening due to a lack of evidence that it independently improves 
patient outcomes. ${ }^{5}$ Furthermore, it has been postulated that the adoption of a standardized cognitive screening process would not only result in earlier identification of dementia but also provide impetus for care planning (including caregiver education, training, and support) to improve patients' overall healthcare and outcomes. ${ }^{6}$

Embedding a process of screening older people for cognitive impairment in routine healthcare settings has the potential to improve care by increasing detection of dementia and prompting development of an individualized care plan for patients and family caregivers. Although other cognitive screening tests such as the Montreal Cognitive Assessment (MoCA) offer greater sensitivity, the Mini-Cog (MC) was chosen for this study because of its brevity, simple administration and scoring procedures expected to minimally impact clinic flow, and accessibility to patients with low education and English as a second language, as well as its sensitivity and specificity in identifying individuals with cognitive impairment in population and clinical studies. ${ }^{7,8}$

Detection of cognitive impairment by itself will have no benefit unless healthcare providers modify their medical decision-making based upon a positive screen result. Two previous studies, one in a community primary care practice in Washington ${ }^{9}$ and the other in a Minnesota-based neurology specialty practice, ${ }^{10}$ evaluated provider response to a positive screening result using a few simple indicators extracted from electronic medical records (EMR). Both studies found that screening increased provider-initiated actions relevant to cognitive impairment, but only a minority of clinicians followed through with a dementia work-up or prescription treatments. However, no studies to our knowledge have addressed healthcare utilization patterns as a function of screendetected cognitive impairment in the ambulatory setting.

In the present study, conducted in an integrated healthcare organization, we examine how often screen-detected cognitive impairment is followed by a dementia work-up and dementiaspecific pharmacotherapy, and compare responses of primary care and neurology specialty care providers. In addition, we compared healthcare utilization during the 18 month pre- and post-testing period between the screen positives and negatives. Our two key hypotheses were (1) cognitive screening would lead to increased dementia diagnosis and management and (2) screening would lead to reduced healthcare utilization, especially acute care episodes, suggesting a shift toward more comprehensive care and preventive interventions once cognitive impairment was recognized.

\section{METHODS}

\section{Population and Setting}

The study sample included patients aged 65 and older who were previously asymptomatic for cognitive impairment and completed brief cognitive screening between August 2011 and June 2014 as part of their visit to select specialty and primary care clinics across the HealthPartners system. HealthPartners is an integrated healthcare organization based in the Twin Cities of Minnesota that provides both healthcare services and health insurance coverage to its more than one million patients and members. Screening was implemented as a pilot quality improvement initiative to increase detection of dementia across the organization. The program was first implemented in a general neurology clinic, where screening was administered by nursing staff to all patients without a known history of cognitive impairment or dementia. The process was subsequently rolled out to four pilot sites in primary care. In the primary care clinics, screening took place in the context of the Medicare Annual Wellness visit (AWV). This study was approved by the HealthPartners Institutional Review Board (IRB).

\section{Data Collection Procedures}

The Mini-Cog is a brief 3-min cognitive screening tool that combines a 3-word recall task with a clock drawing test used as the recall distractor. A total score is derived by summing points for correct word recall (0-3) and clock drawing (0 or 2). The $\mathrm{MC}$ has been validated against research-based diagnostic evaluations for dementia. ${ }^{8}, 7$ Screening positive on the $\mathrm{MC}$ is associated with risk of poor medication management and inability to fill a pillbox correctly ${ }^{11}$ and many other clinically relevant outcomes in medical and surgical patients (see bibliography available at mini-cog.com). Originally, scores of $<3$ best distinguished between patients with and without dementia, but higher cutpoints $(<4)$ have also been used by our group and others to detect cognitive impairment in earlier stages. ${ }^{10,12}$ In addition, the original $\mathrm{MC}<3$ cutpoint with $76 \%$ sensitivity in a population sample is not sufficient to adequately capture all clinically relevant dementia in the population. ${ }^{8}$ Therefore, for this study, we wished to maximize sensitivity and were less concerned about loss of specificity at the higher cutpoint. We therefore chose as the primary independent variable a positive MC defined as a score $<4 / 5$ but conducted a secondary analysis using the traditional $\mathrm{MC}<3$ cut point.

\section{Implementation}

During the start-up phase of the study, nurses in primary care and neurology clinics were trained in MC administration, scoring, and interpretation by a clinical neuropsychologist with expertise in dementia. Paper forms were distributed to all exam rooms at each site and electronic versions of the $\mathrm{MC}$ were imbedded into the electronic medical record system to be printed. Forms included scripting to assist nurses in introducing the task and addressing patient questions. In the neurology clinic, nurses identified eligible patients (aged $\geq 65$ and no history of cognitive impairment or dementia). In primary care clinics, nurses screened all Medicare patients coming in for an annual wellness visit meeting the same criteria. MC scores were reported to the healthcare provider who decided whether further steps should be taken. Several EMR-based decision 
support tools were also developed and available to guide the work-up, diagnosis, and care of patients with cognitive impairment.

Outcomes included MC screen-positive rates, provider follow-up actions based upon items included in our decision support tools, and healthcare utilization for all participants over a period of 36 months (18 months prior to and following MC screening). Provider actions captured included administration of more detailed cognitive screening measures (e.g., MMSE or MoCA), referral for neuropsychological testing, ordering of neuroimaging, referral to a dementia subspecialty clinic, entering of dementia/MCI diagnosis into the EMR, and prescription of a dementia-specific medication (cholinesterase inhibitor or memantine). Healthcare utilization outcomes from insurance claims data included ambulatory, emergency room, and inpatient visits; phone encounters; prescriptions filled; appointment cancelations; and no-shows. Data extraction from the EMR and claims was automated by a study programmer. All subjects were patients and also insured by HealthPartners.

\section{Data Analyses}

An a priori power calculation showed that with 950 subjects with a $20-35 \%$ screen-positive rate, we would have $90 \%$ power to detect a difference of $25 \%$ in the hazard rate for the utilization outcomes (Poisson regression analysis, two-sided, alpha $=0.05$ ). Preliminary analysis included comparing subjects in each group with respect to demographic variables such as age, gender, race, and the presence or absence of three common chronic conditions. These comparisons were conducted using equal variance $t$ tests, chi-square tests, and Fisher's exact tests, as appropriate.

Poisson regression was used to model the monthly rates of healthcare utilization. These models were adjusted for repeated within-person measures. We calculated and tested the significance of multiplicative change in utilization rates between pre- and post-screening time periods as well as between pass and fail groups. Provider behaviors in response to the MC screen results were summarized and compared using chisquare and Fisher exact tests. Additionally, we examined the data at the person-level to determine the number of dementiarelated provider actions completed (of five possible) as an index of intensity of care specific to cognitive impairment. Finally, we further explored the first dementia diagnosis given and first dementia medication prescribed after a positive screen result. This information was extracted from the EMR, and expressed quantitatively, as the mean and median time-todiagnosis and time-to-prescription.

All statistical analyses were conducted in SAS Version 9.4 (SAS Institute, Cary, NC).

\section{RESULTS}

A total of 787 patients (316 [40.2\%] men, 471 [59.8\%] women; average age $77.2 \pm 6.2$ years) were screened for cognitive impairment (331 in a primary care clinic and 456 in a neurology clinic) during the 36-month period (Table 1). The population undergoing screening was demographically representative of the HealthPartners patient population. The screenpositive rate $(\mathrm{MC}<4)$ was $29.3 \%$ with higher rates in neurology $(34.4 \%)$ as compared to primary care $(22.4 \%)$ populations. In addition, individuals screening positive tended to be older, male, and non-white or Hispanic. Analysis of the $\mathrm{MC}<$ 3 cutpoint showed an overall screen-positive rate of $14.5 \%$ (18.0\% neurology; $9.7 \%$ primary care).

We then evaluated the impact of MC results on healthcare provider action. Among patients screening positive on the MC, more detailed cognitive screening (e.g., MMSE or MOCA) was performed in $13.9 \%$, referral for neuropsychological testing made for $3.9 \%$, orders for neuroimaging placed for $3.5 \%$, and referral to a dementia subspecialty clinic initiated in $4.3 \%$ (Table 2). A diagnosis of dementia or MCI was entered into the EMR for $27.3 \%$ of patients after screening positive on the MC while dementia-specific medications (cholinesterase inhibitor or memantine) were prescribed in $10 \%$ of those failing the screen. Compared to those screening negative, patients screening positive on the MC were four times as likely to have additional screening (e.g., MMSE or MOCA), four times more likely to be referred for neuropsychological testing, seven times more likely to have neuroimaging ordered, three times more likely to be referred to a dementia subspecialty clinic, four times more likely to receive a new diagnosis of dementia/MCI, and nine times as likely to receive a dementia-related medication. Although screening positive on the $\mathrm{MC}$ influenced decision-making related to dementia evaluation/management, nearly two thirds of screen-positive individuals $(64.9 \%)$ received no apparent dementia-relevant action (Table 2). Of the five possible dementia-related actions we measured, a single follow-up action was performed in 19.5\% of patients and two follow-up actions were performed in 7.8\% of patients. Otherwise, three or more actions occurred in fewer than $5 \%$ of individuals screening positive.

When comparing healthcare utilization between groups screening positive versus negative (inclusive of both primary care and neurology) during the 18 months pre- and postcognitive screening, subjects screening positive had significantly higher rates of ambulatory visits, ED visits, inpatient hospitalizations, phone encounters, canceled appointments, and no-shows compared to those passing the MC (Table 3). Acute care episodes were more frequent in the screen-positive versus -negative groups during the 36 months surrounding screening: ED visits were $32 \%$ greater during both periods ( $p<0.05$ pre-screen; $p<0.01$ post-screen), and inpatient hospitalizations, $39 \%$ higher in screen-positive individuals during the pre-screening period $(p<0.05)$ and $58 \%$ higher during the post-screening period $(p<0.01)$. Clinic no-shows were $88 \%$ higher before screening $(p<0.01)$ and $38 \%$ higher afterward $(p<0.01)$.

Comparisons of healthcare utilization pre and postscreening among individuals with cognitive impairment 
Table 1 Demographics of Patients Screened

\begin{tabular}{|c|c|c|c|c|c|c|c|}
\hline \multirow[t]{2}{*}{ Characteristic } & \multirow[t]{2}{*}{ Total } & \multicolumn{3}{|c|}{ Positive MC $(<4)$} & \multicolumn{3}{|c|}{ Negative MC (4-5) } \\
\hline & & Total & PC & Neuro & Total & PC & Neuro \\
\hline$N(\%)$ & 787 & $231(29.4)$ & $74(22.4)$ & $157(34.4)$ & $556(70.6)$ & 257 (77.6) & $299(65.6)$ \\
\hline Age (SD) & $77.2(6.2)$ & $79.6(6.4)$ & $78.2(6.9)$ & $80.2(6.1)$ & $76.2(5.8)$ & $75.3(6.0)$ & $77.0(5.4)$ \\
\hline Male & $316(40.2)$ & $100(43.3)$ & $26(35.1)$ & $74(47.1)$ & $216(38.9)$ & $96(37.4)$ & $120(40.1)$ \\
\hline White race ${ }^{1}$ & 751 (95.4) & $216(93.5)$ & $71(96.0)$ & $145(92.4)$ & $535(96.2)$ & $251(97.7)$ & $284(95.0)$ \\
\hline Hispanic & $10(1.3)$ & $4(1.7)$ & $0(0)$ & $4(2.6)$ & $6(1.1)$ & $1(0.4)$ & $5(1.7)$ \\
\hline
\end{tabular}

Values listed as $n(\%)$, unless indicated otherwise

${ }^{1}$ Other races include Black or African American $(n=14)$, Asian or Pacific Islander $(n=3)$, American Indian or Alaskan Native $(n=1)$, some other race $(n=4)$, and NA/unknown $(n=14)$

(including both primary care and neurology populations) revealed no significant change in rates of ambulatory visits, ED visits, inpatient hospitalizations, prescriptions filled, phone encounters, canceled appointments, or no-shows during the initial 18 months compared to the final 18 months (Table 3). Analyses using a MC cutpoint $<3$ showed similar results (online supplemental Tables 1 and 2).

\section{DISCUSSION}

This investigation evaluated the impact of a positive screen for cognitive impairment in older adults on provider action in primary care and neurology practices and patient healthcare utilization across a wide range of services. Employing a cutpoint of $<4$ for MC screen positivity, we found that cognitive impairment was common among older adults without a pre-existing cognitive disorder diagnosis, occurring in approximately 1 in 5 patients within the primary care setting and 1 in 3 within the neurology specialty care setting. These results confirm previous reports of high rates of undetected cognitive impairment among older adults. The higher rate of MC screen positivity in neurology $(34.4 \%)$ relative to primary care settings $(22.4 \%)$ is likely explained by higher rates of central nervous system comorbidity (e.g., Parkinson's disease, stroke, multiple sclerosis, epilepsy) affecting cognition in this population. Despite the use of a higher MC cutpoint of $<4$, the screen-positive rate in the primary care population was consistent with cognitive impairment rates reported in the literature (unadjusted rate 19\%). ${ }^{13}$ The MC cutpoint of $<3$ resulted in a screen-positive rate $(14.5 \%)$ that was below what would be expected in this population, suggesting the limited sensitivity of this parameter.

Mini-Cog score is associated with healthcare utilization even at a cutpoint of $<4$, higher than that previously validated for dementia detection. Individuals screening positive on the MC used more healthcare resources in all six of the measured categories, including ED visits and hospitalizations, compared to those screening negative. Thus, the MC may help identify a subgroup of older adults at increased risk for medical complications and higher cost of care.

Based upon our data, we have found that detection of cognitive impairment with the MC did not impact healthcare utilization. However, it should be noted that relevant provider follow-up action occurred in only a third of individuals, indicating that outcomes may have been different if the majority of providers had adopted best management practices.

Several important limitations of the study should also be noted. This investigation began as a quality improvement process to assess the effect of cognitive screening on dementia diagnosis in primary care and neurology clinics; it is neither a randomized clinical trial nor an implementation trial. In addition, this study did not evaluate the factors underlying provider decision-making based upon the MC result. For instance, $27.3 \%$ of patients screening positive were assigned an MCI or dementia diagnosis code, twice the percentage of individuals who had any documented confirmatory cognitive assessment. Perhaps these patients had cognitive impairment that was so obvious that the clinician chose to omit the work-up; more concerning would be an ill-informed leap to diagnosis on the basis of a screening result. In addition, we did not assess comorbidity for either descriptive or comparative purposes, so are unable to comment on the association of number or type of specific chronic conditions with cognitive impairment or healthcare utilization. Another limitation was our inability to

Table 2 Healthcare Provider Behavior After Mini-Cog Screening

\begin{tabular}{llll}
\hline \hline & \multicolumn{2}{l}{ Positive MC (<4) } & \\
\cline { 2 - 4 } & $\begin{array}{l}\text { Positive } \\
\text { screen }\end{array}$ & $\begin{array}{l}\text { Negative } \\
\text { screen }\end{array}$ & $\begin{array}{l}\boldsymbol{p} \\
\text { value }\end{array}$ \\
\hline $\begin{array}{l}\text { Provider behavior } \\
\text { Additional screening (e.g., }\end{array}$ & $32(13.9)$ & $17(3.1)$ & $<$ \\
$\begin{array}{l}\text { MMSE or MoCA) } \\
\text { Neuropsychology testing }\end{array}$ & $9(3.9)$ & $5(0.9)$ & $0.001^{1}$ \\
$\begin{array}{l}\text { referral } \\
\text { Neuroimaging order }\end{array}$ & $8(3.5)$ & $3(0.5)$ & $0.004^{1}$ \\
$\begin{array}{l}\text { (dementia indication) } \\
\text { Referral to dementia }\end{array}$ & $10(4.3)$ & $8(1.4)$ & $0.004^{2}$ \\
$\begin{array}{l}\text { subspecialty clinic } \\
\text { Dementia/MCI diagnosis }\end{array}$ & $63(27.3)$ & $39(7.0)$ & $0.014^{1}$ \\
entered in EMR & & & $<$ \\
Prescription for dementia & $23(10.0)$ & $6(1.1)$ & $<$ \\
medication & & & $0.001^{1}$ \\
$\begin{array}{l}\text { Number of provider } \\
\text { behaviors }\end{array}$ & & & $<$ \\
None & $150(64.9)$ & $501(90.1)$ & $0.001^{2}$ \\
One & $45(19.5)$ & $42(7.6)$ & \\
$\begin{array}{l}\text { Two } \\
\text { Three }\end{array}$ & $18(7.8)$ & $7(1.3)$ & \\
Four & $9(3.9)$ & $3(0.5)$ & \\
Five & $8(3.5)$ & $2(0.4)$ & \\
\hline
\end{tabular}

Values listed as $n(\%)$

${ }^{1}$ Chi-square test for independence, ${ }^{2}$ Fisher's exact test 
Table 3 Healthcare Utilization Before and After Screening (MC Positive <4)

\begin{tabular}{|c|c|c|c|c|c|c|c|}
\hline \multirow[b]{3}{*}{ Healthcare event } & \multicolumn{3}{|c|}{18 months pre-screen } & \multicolumn{3}{|c|}{18 months post-screen } & \multirow{3}{*}{$\begin{array}{l}\text { + screen post/pre ratio } \\
\text { Total }\end{array}$} \\
\hline & \multicolumn{2}{|c|}{ \# of events } & \multirow{2}{*}{$\frac{ \pm \text { ratio }}{\text { Total }}$} & \multicolumn{2}{|c|}{ \# of events } & \multirow{2}{*}{$\frac{ \pm \text { ratio }}{\text { Total }}$} & \\
\hline & Positive & Negative & & Positive & Negative & & \\
\hline Ambulatory visits & 9597 & 20,474 & $1.13 t$ & 9716 & 20,403 & $1.15 \ddagger$ & 1.01 \\
\hline Emergency room visits & 122 & 223 & $1.32 *$ & 141 & 258 & $1.32 \%$ & 1.16 \\
\hline Inpatient hospitalizations & 92 & 159 & $1.39 *$ & 111 & 169 & $1.58 \%$ & 1.21 \\
\hline Prescriptions filled & 2518 & 5694 & $1.06 t$ & 2586 & 5961 & $1.04^{+}$ & 1.03 \\
\hline Phone encounters ${ }^{1}$ & 3094 & 6092 & $1.22 t$ & 3409 & 6529 & $1.26 \%$ & $1.10^{+}$ \\
\hline Canceled appointments ${ }^{1}$ & 1155 & 2286 & $1.22 t$ & 1297 & 2557 & $1.22 \%$ & 1.12 \\
\hline No-shows ${ }^{1}$ & 217 & 278 & $1.88 \%$ & 223 & 390 & $1.38 \%$ & 1.03 \\
\hline
\end{tabular}

${ }^{1}$ Data from electronic medical record. All other analyses use claims data

${ }^{2}$ Calculated based on the formula: \# of post-screen-positive healthcare events/\# of pre-screen-positive healthcare events

$+p<0.10 ; * p<0.05 ; \neq p<0.01$

track referrals to community-based organizations such as the Alzheimer's Association for care consultation, education, and support, an intervention that would reflect the clinician's appraisal that a positive screen for cognitive impairment is an important finding. Finally, we did not manually review narrative medical records, which could result in underestimation of healthcare utilization as well as provider interventions such as counseling.

A standardized approach to the diagnosis and management of individuals with cognitive impairment is necessary to realize the potential of screening to improve patient care. Multiple healthcare systems throughout the country now screen older adults for cognitive impairment, through either routine clinical visits or the Medicare AWV. The rationale behind this clinical initiative is to facilitate early detection of cognitive impairment as a health risk that should be considered as part of clinical and preventive management. The Centers for Medicare and Medicaid Services introduced a new Medicare benefit in January 2017 that reimburses providers for conducting a multidimensional assessment of individuals with cognitive impairment and developing a care plan that includes engagement of their caregivers. ${ }^{14}$ While this care planning benefit does not require a full differential diagnostic evaluation, we strongly advocate pairing a standardized approach to dementia evaluation and care with any abnormal cognitive screening results or other indicator of possible impairment. Several healthcare systems, including HealthPartners, have developed decision support and management protocols that can be employed throughout the medical system to guide providers in the appropriate workup, diagnosis, and management of affected individuals. Only by supplementing detection and diagnosis with a comprehensive care plan can the value of cognitive screening for improving health outcomes be properly assessed.

Our investigation supports the conclusion that cognitive screening can increase recognition and diagnosis of cognitive disorders in older adults in both primary care and specialty care clinics. Whether or not cognitive screening can improve the quality of healthcare, optimize patterns of healthcare utilization, or improve clinical outcomes are questions that will remain unanswered until provider response to screen-detected impairment becomes standardized. Based upon extensive research over several decades, guidelines have emerged for treatment of several other prevalent chronic conditions such as hypertension, diabetes, hyperlipidemia, and depression. These guidelines in turn have fostered research clearly demonstrating the health benefits of screening for these diseases. A sequence of steps from detection to diagnosis and postdiagnostic care should define a "dementia-capable" healthcare system and provide the essential platform for the much-needed data on how to improve clinical outcomes for cognitively impaired patients.

Corresponding Author: Michael Rosenbloom, MD; Health Partners Center for Memory and Aging, St. Paul, MN, USA (e-mail: Michael.H. Rosenbloom@HealthPartners.Com).

\section{Compliance with Ethical Standards:}

Conflict of Interest: All authors of this manuscript declare that they have no conflicts of interest.

\section{REFERENCES}

1. Association, A. s. (2018). 2018 Alzheimer's disease facts and figures. Alzheimer's \& Dementia, 14(3), 367-429.

2. Kotagal, V., Langa, K. M., Plassman, B. L., Fisher, G. G., Giordani, B. J., Wallace, R. B., . . . Foster, N. L. (2015). Factors associated with cognitive evaluations in the United States. Neurology, 84(1), 64-71. doi:https://doi.org/10.1212/WNL.0000000000001096

3. Milby, E., Murphy, G., \& Winthrop, A. (2017). Diagnosis disclosure in dementia: understanding the experiences of clinicians and patients who have recently given or received a diagnosis. Dementia (London), 16(5), 611-628. doi:https://doi.org/10.1177/1471301215612676

4. Cordell, C. B., Borson, S., Boustani, M., Chodosh, J., Reuben, D., Verghese, J., . . . Fried, L. B. (2013). Alzheimer's Association recommendations for operationalizing the detection of cognitive impairment during the Medicare Annual Wellness Visit in a primary care setting. Alzheimers Dement, 9(2), 141-150. doi:https://doi.org/10.1016/j.jalz. 2012.09.011

5. Lin, J. S., O'Connor, E., Rossom, R. C., Perdue, L. A., Burda, B. U., Thompson, M., \& Eckstrom, E. (2013) Screening for cognitive impairment in older adults: an evidence update for the U.S. Preventive Services Task Force. Rockville (MD).

6. Borson, S., Frank, L., Bayley, P. J., Boustani, M., Dean, M., Lin, P. J., ... Ashford, J. W. (2013). Improving dementia care: the role of screening and detection of cognitive impairment. Alzheimers Dement, 9(2), 151-159. doi:https://doi.org/10.1016/j.jalz.2012.08.008 
7. Borson, S., Scanlan, J., Brush, M., Vitaliano, P., \& Dokmak, A. (2000) The mini-cog: a cognitive 'vital signs' measure for dementia screening in multi-lingual elderly. Int $J$ Geriatr Psychiatry, 15(11), 1021-1027.

8. Borson, S., Scanlan, J. M., Chen, P., \& Ganguli, M. (2003). The MiniCog as a screen for dementia: validation in a population-based sample. $J$ Am Geriatr Soc, 51(10), 1451-1454.

9. Borson, S., Scanlan, J., Hummel, J., Gibbs, K., Lessig, M., \& Zuhr, E. (2007). Implementing routine cognitive screening of older adults in primary care: process and impact on physician behavior. $J$ Gen Intern Med, 22(6), 811-817. doi:https://doi.org/10.1007/s11606-007-0202-8

10. Rosenbloom, M., Borson, S., Barclay, T., Hanson, L. R., Werner, A. Stuck, L., \& McCarten, J. (2016). Routine cognitive screening in a neurology practice: effect on physician behavior. Neurol Clin Pract, 6(1), 16-21. doi:https://doi.org/10.1212/CPJ.0000000000000186

11. Anderson, K., Jue, S. G., \& Madaras-Kelly, K. J. (2008). Identifying patients at risk for medication mismanagement: using cognitive screens to predict a patient's accuracy in filling a pillbox. Consult Pharm, 23(6), 459-472.

12. McCarten, J. R., Anderson, P., Kuskowski, M. A., McPherson, S. E., \& Borson, S. (2011). Screening for cognitive impairment in an elderly veteran population: acceptability and results using different versions of the Mini-Cog. J Am Geriatr Soc, 59(2), 309-313. doi:https://doi.org/10. 1111/j.1532-5415.2010.03249.x

13. Rodríguez-Sánchez, E., Mora-Simón, S., Patino-Alonso, M. C., GarcíaGarcía, R., Escribano-Hernández, A., Garcia-Ortiz, L., ... GómezMarcos, M. A. (2011). Prevalence of cognitive impairment in individuals aged over 65 in an urban area: DERIVA study. BMC Neurology, 11(1), 147.

14. Borson, S., Chodosh, J., Cordell, C., Kallmyer, B., Boustani, M. Chodos, A., ... Thies, w. (2017). Innovation in care for individuals with cognitive impairment: can reimbursement policy spread best practices? Alzheimers Dement, 13(10), 1168-1173. doi:https://doi.org/10.1016/j. jalz.2017.09.001 